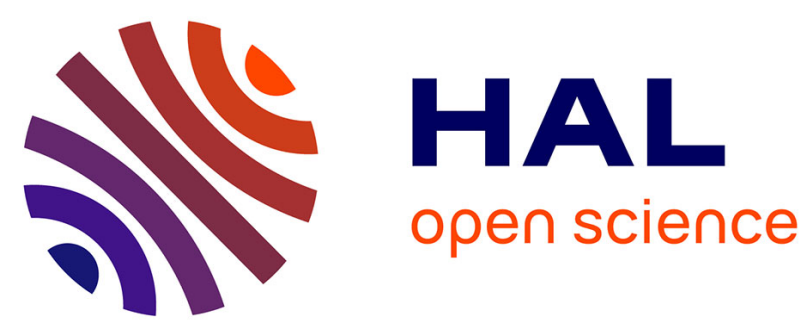

\title{
Weiss-Weinstein bound for MIMO radar with colocated linear arrays for SNR threshold prediction
}

Duy Tran Nguyen, Alexandre Renaux, Remy Boyer, Sylvie Marcos, Pascal Larzabal

\section{- To cite this version:}

Duy Tran Nguyen, Alexandre Renaux, Remy Boyer, Sylvie Marcos, Pascal Larzabal. Weiss-Weinstein bound for MIMO radar with colocated linear arrays for SNR threshold prediction. Signal Processing, 2012, 92 (5), pp.1353-1358. 10.1016/j.sigpro.2011.10.018 . hal-00771395

\section{HAL Id: hal-00771395}

https://hal-centralesupelec.archives-ouvertes.fr/hal-00771395

Submitted on 15 Mar 2020

HAL is a multi-disciplinary open access archive for the deposit and dissemination of scientific research documents, whether they are published or not. The documents may come from teaching and research institutions in France or abroad, or from public or private research centers.
L'archive ouverte pluridisciplinaire $\mathbf{H A L}$, est destinée au dépôt et à la diffusion de documents scientifiques de niveau recherche, publiés ou non, émanant des établissements d'enseignement et de recherche français ou étrangers, des laboratoires publics ou privés. 


\title{
Weiss-Weinstein Bound for MIMO Radar with colocated linear arrays for SNR threshold prediction
}

Nguyen Duy TRAN ${ }^{\mathrm{a}}$, Alexandre RENAUX ${ }^{\mathrm{a}}$, Rémy BOYER ${ }^{\mathrm{a}}$, Sylvie MARCOS ${ }^{\mathrm{a}}$, Pascal LARZABAL

${ }^{a}$ University Paris-Sud 11, Laboratory of Signals and Systems, Supélec, 3 rue Joliot-Curie, 91192 Gif-sur-Yvette, France

${ }^{b}$ Ecole Normale Supérieure de Cachan, SATIE Laboratory, 61 Avenue du Président Wilson, 94235 Cachan Cedex, France

\begin{abstract}
Several works have suggested that a Multi-Input Multi-Output (MIMO) radar system offers improvement in terms of performance in comparison with classical phased-array radar. However, under the widely spread assumption of a uniform a priori distribution for one parameter of interest, there is no result concerning lower bounds on the mean-square error in the case of a Gaussian observation model with parameterized mean. This Fast Communication fills this lack by using the Weiss-Weinstein bound (WWB) which can be calculated under this difficult scenario. A closed-form expression of the WWB for MIMO radar with colocated linear arrays is derived and analyzed for a design purpose in terms of array geometry. Simulations confirm the good ability of the proposed bound to predict the mean square error (MSE) of the maximum $a$ posteriori (MAP) in all range of SNR. Particularly, the tightness of the bound to predict the SNR threshold effect is shown.
\end{abstract}

Keywords: MIMO radar, Weiss-Weinstein bound (WWB), parameter estimation.

\section{Introduction}

Since it was first introduced [1], the MIMO radar has gained interest in investigation to improve multisensor systems performance. A MIMO radar utilizes multiple sensors in both the transmitter and the receiver to send out and collect a set of probing waveforms which can be fully uncorrelated or partially correlated. If all the transmitted waveforms are correlated, it refers to the conventional phased-array radar. Thanks to these additional degrees of freedom, a MIMO radar has many advantages in comparison with a phased-array radar. Depending on the distance between elements of antennas and the positions of the transmitting and receiving arrays (colocated or widely separated), each configuration of MIMO radar has its own interests such as improving parameter identifiability [2] [3]; offering higher flexibility in transmit beam pattern designs: improving the angular resolution, lowering sidelobes, or decreasing the spatial power density of transmitted signals [2]; increasing detection performance, and allowing direct applicability of

This project was funded by both région Île-de-France and Digiteo Research Park. 
adaptive techniques for parameter estimation [4].

If we focus on target localization, the superiority of MIMO radar's performance w.r.t. the phasedarray radar has been shown in terms of lower bound on the mean square error (MSE). To the best of our knowledge, all the existing works available in the literature concern only the case of deterministic parameter for the location of the target. In [1], preliminary calculations of the Cramér-Rao bound (CRB) have been introduced. The considered model was the colocated uniform linear array (ULA) for both transmitting and receiving arrays. In [5] and [6], a MIMO radar with widely-separated antennas was studied and the CRB has been calculated for target position and velocity. Besides, Jian Li et al. have investigated the waveform optimization of MIMO radar based on the CRB using colocated ULA transmitting and receiving antennas [7] [8]. While all the above works have exploited the asymptotic region of the MSE over signal-to-noise ratio (SNR) performance, the Barankin bound has been shown to give a SNR threshold approximation in the scenario of colocated circular array configuration [9] [2](chapter 4).

All these aforementioned investigations handle only the cases where the unknown parameters are assumed to be deterministic. Since the support of the parameters was not taken into account, these bounds cannot describe the overall MSE performance. As an alternative to the deterministic case, we propose to handle the problem in the Bayesian framework which will provide a tight lower bound over all the range of SNR and a good prediction of the SNR threshold. The Bayesian bounds deal with the random parameter and presume an a priori probability density function (pdf). Hence, they will allow us to characterize and to predict more completely MIMO radar performance. Note that not all the Bayesian bounds proposed in the literature are able to take into account the case when the parameters of interest are supposed to be uniformly distributed. Therefore, among various types of Bayesian bounds, such as the Ziv-Zakai bound [10], the Bell-SteinbergEphraim-Van Trees bound [11], the Bobrovsky-Zakai bound [12], and the Bayesian Abel bound [13], we concentrate, in this work, on the Weiss-Weinstein bound (WWB) (see [14] and the recent work of Rapoport and Oshman [15] [16]), which can deal with the uniformly distributed prior assumption and is one of the tightest bound of the Weiss and Weinstein family [17] [18]. The scenario under investigation is a MIMO radar estimating the direction-of-arrival (DOA) of a target and the complex radar-cross-section (RCS) in the Swerling 0 case. Our results are derived in the case of linear (possibly non-uniform) colocated arrays at emission and reception.

The rest of this paper is organized as follows: Section 2 presents the general problem setup with colocated linear arrays. In Section 3, we calculate the corresponding WWB matrix for the DOA and the complex RCS and we prove its diagonal structure. In Section 4, simulations are presented to confirm the good ability of WWB to predict the mean square error (MSE) of the maximum a posteriori (MAP) in all range of SNR (asymptotic and threshold regions). Numerical procedure is introduced to analyze the MIMO radar system in terms of antenna geometry. Finally, Section 5 draws the conclusions. 


\section{Problem Setup}

We consider a single-target localization scenario with a MIMO radar system formed with colocated linear (possibly non-uniform) transmitting and receiving arrays with $M$ and $N$ antennas, respectively. Each transmitting antenna sends out a different waveform, which is known. The $N \times 1$ complex received signal is then given by [7]:

$$
\mathbf{y}(t)=\beta \mathbf{b}(\theta) \mathbf{a}^{T}(\theta) \mathbf{x}(t)+\mathbf{n}(t)=\beta \mathbf{C}(\theta) \mathbf{x}(t)+\mathbf{n}(t), t=1 \ldots T
$$

where $T$ is the number of snapshots. Since both transmitting and receiving arrays are linear, the steering vectors have the following forms

$$
\begin{aligned}
& \mathbf{a}(\theta)=\left[\exp \left(-j \frac{2 \pi}{\lambda} a_{1} \sin \theta\right), \ldots, \exp \left(-j \frac{2 \pi}{\lambda} a_{M} \sin \theta\right)\right]^{T}, \\
& \mathbf{b}(\theta)=\left[\exp \left(-j \frac{2 \pi}{\lambda} b_{1} \sin \theta\right), \ldots, \exp \left(-j \frac{2 \pi}{\lambda} b_{N} \sin \theta\right)\right]^{T},
\end{aligned}
$$

where $\lambda$ denotes the wavelength, where $a_{i}, i=1 \ldots M$, and $b_{j}, j=1 \ldots N$, are the positions of the elements (w.r.t. a reference point) of the transmitting and receiving arrays, respectively. Note that for the colocated scenario, the DOA is the same as the direction-of-departure (DOD), hence $\theta$ is the only one location parameter of the target. Moreover, $\theta$ is assumed to have an a priori uniform distribution over the support $[0, \pi]$. For simplicity in the derivation, we define $u=\sin (\theta)$ as the parameter of interest instead of working directly with $\theta$. Note that, the pdf for $u$ is $p(u)=\frac{1}{\pi \sqrt{1-u^{2}}}$. We denote $\mathbf{C}(\theta)=\mathbf{b}(\theta) \mathbf{a}^{T}(\theta) \in \mathbb{C}^{N \times M}$. The elements of $\mathbf{C}(\theta)$ are given by $[\mathbf{C}(\theta)]_{k, l}=\left[\exp \left(-j \frac{2 \pi}{\lambda}\left(a_{l}+b_{k}\right) \sin \theta\right)\right] . \mathbf{x}(t)$ is the vector of each transmitted waveform. We suppose that these waveforms are independent and have the following empirical covariance matrix: $\mathbf{R}_{x}=\frac{1}{T} \sum_{t=1}^{T} \mathbf{x}(t) \mathbf{x}^{H}(t)=\operatorname{Diag}\left(\boldsymbol{\sigma}_{s}^{2}\right)$, where $\boldsymbol{\sigma}_{s}^{2}=\left[\sigma_{1}^{2}, \ldots, \sigma_{M}^{2}\right]^{T}$. We also define $\{\mathbf{n}(t)\}_{t=1}^{T}$ as the noise vectors, which are assumed to be independent and identically distributed circularly complex Gaussian with zero-mean and covariance matrix $\mathbf{R}=\sigma_{n}^{2} \mathbf{I}$. $\beta$ is the target complex amplitude related to the RCS of the target in the Swerling 0 case, and has an a priori circular complex Gaussian distribution with zero mean and variance $\sigma_{\beta}^{2}$, namely $\beta \sim \mathcal{C N}\left(0, \sigma_{\beta}^{2}\right)$. The elements of the unknown parameter vector $\Theta=\left[u, \beta_{R}, \beta_{I}\right]^{T}$ with $\beta_{R}=\operatorname{Re}\{\beta\}, \beta_{I}=\operatorname{Im}\{\beta\}$, are considered to be statistically independent such that the joint pdf $p(\boldsymbol{\Theta})=p(u) p\left(\beta_{R}\right) p\left(\beta_{I}\right)$.

Under the assumption of independent observations, the likelihood of the full set of observations $\mathbf{Y}=$ $[\mathbf{y}(1), \mathbf{y}(2), \ldots, \mathbf{y}(T)]$ is given by

$$
p(\mathbf{Y} ; \boldsymbol{\Theta})=\frac{1}{\pi^{N T} \sigma_{n}^{N T}} \exp \left(-\frac{1}{\sigma_{n}^{2}} \sum_{t=1}^{T}(\mathbf{y}(t)-\beta \mathbf{C}(\theta) \mathbf{x}(t))^{H}(\mathbf{y}(t)-\beta \mathbf{C}(\theta) \mathbf{x}(t))\right) .
$$




\section{Weiss-Weinstein Bound for MIMO radar 's parameter estimation}

The Weiss-Weinstein bound [14], denoted by WWB, of the unknown target parameters $\boldsymbol{\Theta}=\left[u, \beta_{R}, \beta_{I}\right]^{T}$ is a $3 \times 3$ matrix such that the following relation holds

$$
E\left\{(\hat{\mathbf{\Theta}}-\mathbf{\Theta})(\hat{\mathbf{\Theta}}-\boldsymbol{\Theta})^{T}\right\} \geq \mathbf{W W B}=\sup _{\substack{\mathbf{h}_{i}, s_{i} \\ i=1,2,3}} \mathbf{H G}^{-1} \mathbf{H}^{T},
$$

where $E\left\{(\hat{\boldsymbol{\Theta}}-\mathbf{\Theta})(\hat{\boldsymbol{\Theta}}-\mathbf{\Theta})^{T}\right\}$ is the MSE of any Bayesian estimator $\hat{\boldsymbol{\Theta}}$ and $\mathbf{H}=\left[\mathbf{h}_{1}, \mathbf{h}_{2}, \mathbf{h}_{3}\right]$ is the $3 \times 3$ matrix of test-points. The elements of the $3 \times 3$ matrix $\mathbf{G}$ are given by

$$
\mathbf{G}_{k l}=\frac{E\left\{\left[L^{s_{k}}\left(\mathbf{Y} ; \boldsymbol{\Theta}+\mathbf{h}_{k}, \boldsymbol{\Theta}\right)-L^{1-s_{k}}\left(\mathbf{Y} ; \boldsymbol{\Theta}-\mathbf{h}_{k}, \boldsymbol{\Theta}\right)\right]\left[L^{s_{l}}\left(\mathbf{Y} ; \boldsymbol{\Theta}+\mathbf{h}_{l}, \boldsymbol{\Theta}\right)-L^{1-s_{l}}\left(\mathbf{Y} ; \boldsymbol{\Theta}-\mathbf{h}_{l}, \boldsymbol{\Theta}\right)\right]\right\}}{E\left\{L^{s_{k}}\left(\mathbf{Y} ; \boldsymbol{\Theta}+\mathbf{h}_{k}, \boldsymbol{\Theta}\right)\right\} E\left\{L^{s_{l}}\left(\mathbf{Y} ; \boldsymbol{\Theta}+\mathbf{h}_{l}, \boldsymbol{\Theta}\right)\right\}}
$$

where $L\left(\mathbf{Y} ; \boldsymbol{\Theta}+\mathbf{h}_{i}, \boldsymbol{\Theta}\right)=\frac{p\left(\mathbf{Y}, \boldsymbol{\Theta}+\mathbf{h}_{i}\right)}{p(\mathbf{Y}, \boldsymbol{\Theta})}$, and $s_{i} \in[0,1], i=1,2,3$. The expectations are taken over the joint $\operatorname{pdf} p(\mathbf{Y}, \boldsymbol{\Theta})$.

Rigorously, the quantity $\mathbf{H G}^{-1} \mathbf{H}^{T}$ must be maximized w.r.t. $\mathbf{h}_{i}$ and $s_{i}$ leading to a high computational complexity. However, it has been shown [19] [20] that choosing $s_{i}=\frac{1}{2}, \forall i=1,2,3$, and a diagonal matrix $\mathbf{H}$ leads to a bound still tight. Therefore, we assume here that $s_{i}=1 / 2, \forall i$, and that $\mathbf{H}=\operatorname{Diag}\left(\left[h_{1}, h_{2}, h_{3}\right]^{T}\right)$. In this case, the elements of the matrix $\mathbf{G}$ can be written as

$$
\mathbf{G}_{k l}=\frac{\eta\left(\mathbf{h}_{k}, \mathbf{h}_{l}\right)+\eta\left(-\mathbf{h}_{k},-\mathbf{h}_{l}\right)-\eta\left(\mathbf{h}_{k},-\mathbf{h}_{l}\right)-\eta\left(-\mathbf{h}_{k}, \mathbf{h}_{l}\right)}{\eta\left(\mathbf{h}_{k}, \mathbf{0}\right) \eta\left(\mathbf{h}_{l}, \mathbf{0}\right)},
$$

where we define

$$
\eta(\boldsymbol{\alpha}, \boldsymbol{\gamma})=\int_{\Upsilon} \int_{\boldsymbol{\Omega}} p(\mathbf{Y}, \boldsymbol{\Theta}+\boldsymbol{\alpha})^{\frac{1}{2}} p(\mathbf{Y}, \mathbf{\Theta}+\boldsymbol{\gamma})^{\frac{1}{2}} d \mathbf{Y} d \mathbf{\Theta}
$$

where $\boldsymbol{\Omega}$ and $\boldsymbol{\Upsilon}$ are the observation and parameter spaces, respectively.

By denoting

$$
\eta^{\prime}(\boldsymbol{\Theta}, \boldsymbol{\alpha}, \boldsymbol{\gamma})=\int_{\boldsymbol{\Omega}} p(\mathbf{Y} ; \boldsymbol{\Theta}+\boldsymbol{\alpha})^{\frac{1}{2}} p(\mathbf{Y} ; \boldsymbol{\Theta}+\boldsymbol{\gamma})^{\frac{1}{2}} d \mathbf{Y}
$$

$\eta(\boldsymbol{\alpha}, \boldsymbol{\gamma})$ can be rewritten as

$$
\eta(\boldsymbol{\alpha}, \boldsymbol{\gamma})=\int_{\boldsymbol{\Upsilon}} \eta^{\prime}(\boldsymbol{\Theta}, \boldsymbol{\alpha}, \boldsymbol{\gamma}) p(\boldsymbol{\Theta}+\boldsymbol{\alpha})^{\frac{1}{2}} p(\boldsymbol{\Theta}+\boldsymbol{\gamma})^{\frac{1}{2}} d \boldsymbol{\Theta} .
$$

Plugging (4) into (9), we obtain a closed-form expression for $\eta^{\prime}$ (the details are given in Appendix 6.1)

$$
\eta^{\prime}(\boldsymbol{\Theta}, \boldsymbol{\alpha}, \boldsymbol{\gamma})=\exp \left(-\frac{1}{4 \sigma_{n}^{2}} \sum_{t=1}^{T}[\mathbf{f}(\boldsymbol{\Theta}+\boldsymbol{\alpha}, t)-\mathbf{f}(\boldsymbol{\Theta}+\boldsymbol{\gamma}, t)]^{H}[\mathbf{f}(\boldsymbol{\Theta}+\boldsymbol{\alpha}, t)-\mathbf{f}(\boldsymbol{\Theta}+\boldsymbol{\gamma}, t)]\right),
$$


where $\mathbf{f}(\boldsymbol{\Theta}, t)=\beta \mathbf{C}(\theta) \mathbf{x}(t)$. Next, an integration over the support of the parameters is required in order to derive $\eta$. Note that, if we assume a uniform a priori pdf on $\theta$, the pdf for $u$ is $p(u)=\frac{1}{\pi \sqrt{1-u^{2}}}$ which causes an analytical development of the required integration unobtainable. This leads to a numerical integration to calculate the considering bound which is very time-consuming. Moreover, a closed-form expression is always interesting because it allows an easy interpretation of the bound, i.e., we can study the performance of the MIMO radar system w.r.t. some design parameters. Consequently, with the intention of obtaining closed-form expression of WWB, we propose a different approach where we assume a uniform a priori pdf on $u$ (see also [21] where the same thing appears in the context of classical bearing estimation). Moreover, whatever the a priori pdf on $u$, it is theoretically proved that we still obtain a strict bound on the MSE. We also show by simulation (in Section 4.2) that the two approaches give very close bounds and the same prediction of the SNR threshold.

Assuming a uniform a priori pdf on $u$, the structure of the matrix WWB is given as follows (see Appendix 6.2 for more details):

$$
\mathbf{W W B}=\operatorname{Diag}\left(\sup _{h_{1}} \frac{h_{1}^{2}}{\mathbf{G}_{11}}, \frac{1}{2\left(T A S N R+\frac{1}{\sigma_{\beta}^{2}}\right)}, \frac{1}{2\left(T A S N R+\frac{1}{\sigma_{\beta}^{2}}\right)}\right),
$$

where the array signal to noise $(A S N R)$ is defined as $P N / \sigma_{n}^{2}$ where $P=\sum_{k=1}^{M} \sigma_{k}^{2}$ is the total transmitted power, and where $\mathbf{G}_{\mathbf{1 1}}$ is given by

$$
\mathbf{G}_{11}=4 \frac{\left[\left(2-h_{1}\right) f\left(2, h_{1}\right)-2\left(1-h_{1}\right)\right] f\left(1, h_{1}\right)^{2}}{\left(2-h_{1}\right)^{2} f\left(2, h_{1}\right)},
$$

where we define

$$
f\left(\varphi, h_{1}\right)=\sigma_{\beta}^{2} \frac{1}{2} T \sum_{k=1}^{M} \sum_{r=1}^{N} \frac{\sigma_{k}^{2}}{\sigma_{n}^{2}}\left[1-\cos \left(\frac{\varphi 2 \pi}{\lambda} h_{1}\left(a_{k}+b_{r}\right)\right)\right]+1 .
$$

In the following, we analyse the proposed bound in terms of SNR threshold prediction and array geometry design.

\section{Analysis}

\subsection{Properties of the bound}

There are three properties of the WWB matrix (12):

1. Due to the diagonal structure of the WWB matrix, the lower bounds on estimation errors are decoupled. 
2. Thanks to this derivation, the initial multidimensional optimization problem w.r.t. $h_{1}, h_{2}$, and $h_{3}$ has been reduced to a monodimensional optimization problem w.r.t. $h_{1}$ only.

3. The WWB for $\beta_{R}$ and $\beta_{I}$ are the same as their Bayesian CRB in the case where $u$ is assumed to be known (the proof is straightforward).

\subsection{WWB performance in predicting the global MSE and the SNR threshold}

The aim of this part is to examine the usefulness of using the WWB to predict the ultimate global MSE and the SNR threshold. For that reason, we consider the empirical global MSE of the MAP estimator, which is evaluated over 1000 Monte Carlo trials. The simulation is performed with parameters as follows: the transmit array is sparse with $M=8$ sensors and with inter-element spacing (in unit of wavelengths) equals to 4 and the receive array is an ULA with $N=8$ sensors and with inter-element spacing (in unit of wavelengths) equals to 0.5. Such array configuration is well-known in MIMO radar ([2] p.76) to create a Nyquist virtual array with good performance. The uncorrelated MIMO radar waveforms are generated using Hadamard codes with $T=64$ snapshots. The transmitted power is assumed to be uniformly distributed on all transmit antennas. $h_{1}$ is chosen on the support $[-1,1]$. Figure 1 shows the WWB and the MAP of the parameter of interest $u$ versus ASNR. It can be seen that the WWB provides a good prediction of the MSE in all regions. It predicts the threshold SNR location to be $4 \mathrm{~dB}$ below the threshold SNR indicated by the MAP.

Next, as aforementioned, there are two possible assumptions for the a priori pdf on $u$, one leads to a numerical integration and another leads to a closed-form expression of the bound. In Figure 2, we show that the two assumptions give very close bounds and the same prediction of the SNR threshold. Note that, in our simulation, time needed to calculate the bound with numerical integration is over 100 times of time in the other case. Consequently, to have a (still tight) bound with efficiently computational cost and easy interpretation, the WWB derived with the assumption of uniform a priori pdf on $u$ is preferred especially for designing MIMO radar system.

\subsection{MIMO array geometry investigation}

This part investigates the behavior of the derived WWB w.r.t. the array geometry of both the receiver and transmitter. Note that the proposed bound has been derived in the context of linear arrays but possibly non-uniform. Consequently, it remains a degree of freedom to enhance the performance. We first analyze the impact of the receiver array geometry while the transmitter is made from a classical ULA. Both transmitter and receiver are made with $M=N=8$ sensors. The transmitter is a fixed ULA (half-wavelength interelement spacing) array. The receiver has different linear array geometries keeping the array aperture $D=23$

(in unit of $\frac{\lambda}{2}$ ) fixed (i.e. the positions of the 2 extreme sensors of the linear array are fixed). In other words, their is 22 positions available for the 6 other sensors (74613 possibilities). The number of observations is 
$T=64$. We have generated all the array configurations and computed the associated WWB. In Figure 3, among these 74613 bounds we have plotted the bound which leads to the lowest SNR threshold (denoted bound A) and the bound which has the lowest MSE in the asymptotic area (denoted bound B). We have noticed that both geometries leading to bound $\mathrm{A}$ and $\mathrm{B}$ are not with minimum redundancy or minimum gap. Indeed, for this configuration, it can be seen that it exists four minimum-redundancy arrays. We have computed the bounds associated to these four possibilities and plotted the best one in terms of MSE for comparison purpose. The bound A (i.e. the bound with the lowest SNR threshold) shows that better asymptotic performance can be obtained. It exists a geometry configuration leading to slightly better asymptotic performance (bound B) but with a worst SNR threshold. Note that, we have obtained the same results, i.e. same bounds associated to the same optimal geometries, in the opposite case where the receiver is a fixed ULA and where the transmitter array geometry is optimized following the same aforementioned way.

\section{Conclusions}

In this correspondence, we have derived a closed-form expression of the Weiss-Weinstein bound for MIMO Radar with colocated linear arrays. It has been seen that the Weiss-Weinstein bound for the parameter of interest provides a good prediction of the MSE in all regions. It also predicts the threshold SNR location near the threshold SNR indicated by the MAP. We have also investigated the influence of array geometry on the behavior of the Weiss-Weinstein bound.

\section{Appendix}

\subsection{Derivation of $\eta^{\prime}(\boldsymbol{\Theta}, \boldsymbol{\alpha}, \gamma)$}

Note that

$$
p(\mathbf{Y} ; \boldsymbol{\Theta}+\boldsymbol{\alpha})^{\frac{1}{2}} p(\mathbf{Y} ; \boldsymbol{\Theta}+\boldsymbol{\gamma})^{\frac{1}{2}}=\frac{1}{\pi^{N T} \sigma_{n}^{N T}} \exp \left(-\sum_{t=1}^{T} \zeta(\boldsymbol{\Theta}, \boldsymbol{\alpha}, \boldsymbol{\gamma}, t)\right)
$$

where

$$
\begin{aligned}
& \zeta(\boldsymbol{\Theta}, \boldsymbol{\alpha}, \boldsymbol{\gamma}, t)=\frac{1}{2 \sigma_{n}^{2}}[\mathbf{y}-\mathbf{f}(\boldsymbol{\Theta}+\boldsymbol{\alpha}, t)]^{H}[\mathbf{y}-\mathbf{f}(\boldsymbol{\Theta}+\boldsymbol{\alpha}, t)]+\frac{1}{2 \sigma_{n}^{2}}[\mathbf{y}-\mathbf{f}(\boldsymbol{\Theta}+\boldsymbol{\gamma}, t)]^{H}[\mathbf{y}-\mathbf{f}(\boldsymbol{\Theta}+\boldsymbol{\gamma}, t)] \\
& =\frac{1}{\sigma_{n}^{2}}\left[\mathbf{y}^{H} \mathbf{y}-\frac{1}{2} \mathbf{y}^{H} \mathbf{f}(\boldsymbol{\Theta}+\boldsymbol{\alpha}, t)-\frac{1}{2} \mathbf{y}^{H} \mathbf{f}(\boldsymbol{\Theta}+\boldsymbol{\gamma}, t)-\frac{1}{2} \mathbf{f}(\boldsymbol{\Theta}+\boldsymbol{\alpha}, t)^{H} \mathbf{y}-\frac{1}{2} \mathbf{f}(\boldsymbol{\Theta}+\boldsymbol{\gamma}, t)^{H} \mathbf{y}\right. \\
& \left.+\frac{1}{2} \mathbf{f}(\boldsymbol{\Theta}+\boldsymbol{\alpha}, t)^{H} \mathbf{f}(\boldsymbol{\Theta}+\boldsymbol{\alpha}, t)+\frac{1}{2} \mathbf{f}(\boldsymbol{\Theta}+\boldsymbol{\gamma}, t)^{H} \mathbf{f}(\boldsymbol{\Theta}+\boldsymbol{\gamma}, t)\right] .
\end{aligned}
$$

Define $\mathbf{z}=\mathbf{y}-\frac{1}{2} \mathbf{f}(\boldsymbol{\Theta}+\boldsymbol{\alpha}, t)-\frac{1}{2} \mathbf{f}(\boldsymbol{\Theta}+\boldsymbol{\gamma}, t)$, we have 


$$
\zeta(\boldsymbol{\Theta}, \boldsymbol{\alpha}, \boldsymbol{\gamma}, t)=\frac{1}{\sigma_{n}^{2}} \mathbf{z}^{H} \mathbf{z}+\frac{1}{4} \zeta^{\prime}(\boldsymbol{\Theta}, \boldsymbol{\alpha}, \boldsymbol{\gamma}, t)
$$

where $\zeta^{\prime}(\boldsymbol{\Theta}, \boldsymbol{\alpha}, \boldsymbol{\gamma}, t)=\frac{1}{\sigma_{n}^{2}}[\mathbf{f}(\boldsymbol{\Theta}+\boldsymbol{\alpha}, t)-\mathbf{f}(\boldsymbol{\Theta}+\boldsymbol{\gamma}, t)]^{H}[\mathbf{f}(\boldsymbol{\Theta}+\boldsymbol{\alpha}, t)-\mathbf{f}(\boldsymbol{\Theta}+\boldsymbol{\gamma}, t)]$. Consequently, $\zeta^{\prime}(\boldsymbol{\Theta}, \boldsymbol{\alpha}, \boldsymbol{\gamma}, t)$ does not depend on $\mathbf{Y}$, and

$$
\begin{aligned}
\eta^{\prime}(\boldsymbol{\Theta}, \boldsymbol{\alpha}, \boldsymbol{\gamma}) & =\frac{1}{\pi^{N T} \sigma_{n}^{N T}} \int_{\Omega} \exp \left(-\sum_{t=1}^{T}\left(\frac{1}{\sigma_{n}^{2}} \mathbf{z}^{H} \mathbf{z}+\frac{1}{4} \zeta^{\prime}(\boldsymbol{\Theta}, \boldsymbol{\alpha}, \boldsymbol{\gamma}, t)\right)\right) d \mathbf{Y} \\
& \left.=\exp \left(-\sum_{t=1}^{T} \frac{1}{4} \zeta^{\prime}(\boldsymbol{\Theta}, \boldsymbol{\alpha}, \boldsymbol{\gamma}, t)\right)\right) .
\end{aligned}
$$

\subsection{Derivation of matrix $\mathbf{W W B}$}

From (18), the set of functions involved in $\mathbf{G}_{11}$ are given by

$$
\left\{\begin{array}{l}
\eta^{\prime}\left(\boldsymbol{\Theta}, \mathbf{h}_{1}, \mathbf{h}_{1}\right)=1 \\
\eta^{\prime}\left(\boldsymbol{\Theta},-\mathbf{h}_{1},-\mathbf{h}_{1}\right)=1 \\
\eta^{\prime}\left(\boldsymbol{\Theta}, \mathbf{h}_{1},-\mathbf{h}_{1}\right)=\exp \left\{-\frac{1}{2}\left(\beta_{R}^{2}+\beta_{I}^{2}\right) T \sum_{k=1}^{M} \sum_{r=1}^{N} \frac{\sigma_{k}^{2}}{\sigma_{n}^{2}}\left[1-\cos \left(\frac{4 \pi}{\lambda} h_{1}\left(a_{k}+b_{r}\right)\right)\right]\right\} \\
\eta^{\prime}\left(\boldsymbol{\Theta}, \mathbf{h}_{1}, 0\right)=\exp \left\{-\frac{1}{2}\left(\beta_{R}^{2}+\beta_{I}^{2}\right) 2 T \sum_{k=1}^{M} \sum_{r=1}^{N} \frac{\sigma_{k}^{2}}{\sigma_{n}^{2}}\left[1-\cos \left(\frac{2 \pi}{\lambda} h_{1}\left(a_{k}+b_{r}\right)\right)\right]\right\} .
\end{array}\right.
$$

Then, since all the functions $\eta^{\prime}$ do not depend on $u$, by integrating w.r.t. $\Theta$, we obtain

$$
\left\{\begin{array}{l}
\eta\left(\mathbf{h}_{1}, \mathbf{h}_{1}\right)=\frac{2-h_{1}}{2}, \\
\eta\left(-\mathbf{h}_{1},-\mathbf{h}_{1}\right)=\frac{2-h_{1}}{2}, \\
\eta\left(\mathbf{h}_{1},-\mathbf{h}_{1}\right)=\frac{1-h_{1}}{\sigma_{\beta}^{2} \frac{1}{2} T \sum_{k=1}^{M} \sum_{r=1}^{N} \frac{\sigma_{k}^{2}}{\sigma_{n}^{2}}\left[1-\cos \left(\frac{4 \pi}{\lambda} h_{1}\left(a_{k}+b_{r}\right)\right)\right]+1}, \\
\eta\left(\mathbf{h}_{1}, 0\right)=\frac{2-h_{1}}{2} \frac{1}{\sigma_{\beta}^{2} \frac{1}{2} T \sum_{k=1}^{M} \sum_{r=1}^{N} \frac{\sigma_{k}^{2}}{\sigma_{n}^{2}}\left[1-\cos \left(\frac{2 \pi}{\lambda} h_{1}\left(a_{k}+b_{r}\right)\right)\right]+1} .
\end{array}\right.
$$

The set of functions involved in $\mathbf{G}_{k k}, k=2,3$ are given by

$$
\left\{\begin{array}{l}
\eta^{\prime}\left(\boldsymbol{\Theta}, \mathbf{h}_{k}, \mathbf{h}_{k}\right)=1 \\
\eta^{\prime}\left(\boldsymbol{\Theta},-\mathbf{h}_{k},-\mathbf{h}_{k}\right)=1 \\
\eta^{\prime}\left(\boldsymbol{\Theta}, \mathbf{h}_{k},-\mathbf{h}_{k}\right)=\exp \left(-h_{k}^{2} N T \frac{\sum_{k=1}^{M} \sigma_{k}^{2}}{\sigma_{n}^{2}}\right) \\
\eta^{\prime}\left(\Theta, \mathbf{h}_{k}, 0\right)=\exp \left(-\frac{1}{4} h_{k}^{2} N T \frac{\sum_{k=1}^{M} \sigma_{k}^{2}}{\sigma_{n}^{2}}\right)
\end{array}\right.
$$

Then,

$$
\left\{\begin{array}{l}
\eta\left(\mathbf{h}_{k}, \mathbf{h}_{k}\right)=1 \\
\eta\left(-\mathbf{h}_{k},-\mathbf{h}_{k}\right)=1 \\
\eta\left(\mathbf{h}_{k},-\mathbf{h}_{k}\right)=\exp \left(-h_{k}^{2} N T \frac{\sum_{k=1}^{M} \sigma_{k}^{2}}{\sigma_{n}^{2}}\right) \exp \left(-\frac{h_{k}^{2}}{\sigma_{\beta}^{2}}\right) \\
\eta\left(\mathbf{h}_{k}, 0\right)=\exp \left(-\frac{1}{4} h_{k}^{2} N T \frac{\sum_{k=1}^{M} \sigma_{k}^{2}}{\sigma_{n}^{2}}\right) \exp \left(-\frac{h_{k}^{2}}{4 \sigma_{\beta}^{2}}\right)
\end{array}\right.
$$


The set of functions involved in $\mathbf{G}_{12}$ are given by

$$
\left\{\begin{array}{r}
\eta^{\prime}\left(\boldsymbol{\Theta}, \mathbf{h}_{1}, \mathbf{h}_{2}\right)=\exp \left\{2 T \sum _ { k = 1 } ^ { M } \sum _ { r = 1 } ^ { N } \frac { \sigma _ { k } ^ { 2 } } { \sigma _ { n } ^ { 2 } } \left[\beta_{R}^{2}\left(1-\cos \left(\frac{2 \pi}{\lambda} h_{1}\left(a_{k}+b_{r}\right)\right)\right)+\beta_{R} h_{2}\left(1-\cos \left(\frac{2 \pi}{\lambda} h_{1}\left(a_{k}+b_{r}\right)\right)\right)+\right.\right. \\
\left.\left.\beta_{I}^{2}\left(1-\cos \left(\frac{2 \pi}{\lambda} h_{1}\left(a_{k}+b_{r}\right)\right)\right)-\beta_{I} h_{2} \sin \left(\frac{2 \pi}{\lambda} h_{1}\left(a_{k}+b_{r}\right)\right)+\frac{1}{2} h_{2}^{2}\right]\right\} \\
\eta^{\prime}\left(\boldsymbol{\Theta},-\mathbf{h}_{1},-\mathbf{h}_{2}\right)=\exp \left\{2 T \sum _ { k = 1 } ^ { M } \sum _ { r = 1 } ^ { N } \frac { \sigma _ { k } ^ { 2 } } { \sigma _ { n } ^ { 2 } } \left[\beta_{R}^{2}\left(1-\cos \left(\frac{2 \pi}{\lambda} h_{1}\left(a_{k}+b_{r}\right)\right)\right)-\beta_{R} h_{2}\left(1-\cos \left(\frac{2 \pi}{\lambda} h_{1}\left(a_{k}+b_{r}\right)\right)\right)+\right.\right. \\
\left.\left.\beta_{I}^{2}\left(1-\cos \left(\frac{2 \pi}{\lambda} h_{1}\left(a_{k}+b_{r}\right)\right)\right)+\beta_{I} h_{2} \sin \left(\frac{2 \pi}{\lambda} h_{1}\left(a_{k}+b_{r}\right)\right)+\frac{1}{2} h_{2}^{2}\right] \exp \right\} \\
\eta^{\prime}\left(\boldsymbol{\Theta},-\mathbf{h}_{1}, \mathbf{h}_{2}\right)=\exp \left\{2 T \sum _ { k = 1 } ^ { M } \sum _ { r = 1 } ^ { N } \frac { \sigma _ { k } ^ { 2 } } { \sigma _ { n } ^ { 2 } } \left[\beta_{R}^{2}\left(1-\cos \left(\frac{2 \pi}{\lambda} h_{1}\left(a_{k}+b_{r}\right)\right)\right)+\beta_{R} h_{2}\left(1-\cos \left(\frac{2 \pi}{\lambda} h_{1}\left(a_{k}+b_{r}\right)\right)\right)+\right.\right. \\
\left.\left.\beta_{I}^{2}\left(1-\cos \left(\frac{2 \pi}{\lambda} h_{1}\left(a_{k}+b_{r}\right)\right)\right)+\beta_{I} h_{2} \sin \left(\frac{2 \pi}{\lambda} h_{1}\left(a_{k}+b_{r}\right)\right)+\frac{1}{2} h_{2}^{2}\right] \exp \right\} \\
\eta^{\prime}\left(\boldsymbol{\Theta},-\mathbf{h}_{1},-\mathbf{h}_{2}\right)=\exp \left\{2 T \sum _ { k = 1 } ^ { M } \sum _ { r = 1 } ^ { N } \frac { \sigma _ { k } ^ { 2 } } { \sigma _ { n } ^ { 2 } } \left[\beta_{R}^{2}\left(1-\cos \left(\frac{2 \pi}{\lambda} h_{1}\left(a_{k}+b_{r}\right)\right)\right)-\beta_{R} h_{2}\left(1-\cos \left(\frac{2 \pi}{\lambda} h_{1}\left(a_{k}+b_{r}\right)\right)\right)+\right.\right. \\
\left.\left.\beta_{I}^{2}\left(1-\cos \left(\frac{2 \pi}{\lambda} h_{1}\left(a_{k}+b_{r}\right)\right)\right)-\beta_{I} h_{2} \sin \left(\frac{2 \pi}{\lambda} h_{1}\left(a_{k}+b_{r}\right)\right)+\frac{1}{2} h_{2}^{2}\right] \exp \right\} .
\end{array}\right.
$$

Consequently,

$$
\eta\left(\mathbf{h}_{1}, \mathbf{h}_{2}\right)=\eta\left(-\mathbf{h}_{1}, \mathbf{h}_{2}\right)=\frac{2-h_{1}}{2} t_{1} t_{2}
$$

where

$$
\begin{aligned}
t_{1}= & \exp \left(-\frac{h_{2}^{2}}{2 \sigma_{\beta}^{2}}\right) \exp \left\{\frac{h_{2}^{2}}{4}\left[\frac{T}{2} \sum_{k=1}^{M} \sum_{r=1}^{N} \frac{\sigma_{k}^{2}}{\sigma_{n}^{2}}\left(1-\cos \left(\frac{2 \pi}{\lambda} h_{1}\left(a_{k}+b_{r}\right)\right)+\frac{1}{\sigma_{\beta}^{2}}\right]\right\}\right. \\
& \times \frac{\sqrt{\pi} \sigma_{\beta}}{4\left\{\frac{\sigma_{\beta}^{2}}{2}\left[\frac{T}{2} \sum_{k=1}^{M} \sum_{r=1}^{N} \frac{\sigma_{k}^{2}}{\sigma_{n}^{2}}\left(1-\cos \left(\frac{2 \pi}{\lambda} h_{1}\left(a_{k}+b_{r}\right)\right)\right)+\frac{1}{\sigma_{\beta}^{2}}\right]\right\}^{\frac{1}{2}}} \\
t_{2}= & \exp \left\{\frac{\left[h_{2} \frac{T}{2} \sum_{k=1}^{M} \sum_{r=1}^{N} \frac{\sigma_{k}^{2}}{\sigma_{n}^{2}} \sin \left(\frac{2 \pi}{\lambda} h_{1}\left(a_{k}+b_{r}\right)\right)\right]^{2}}{4\left[\frac{T}{2} \sum_{k=1}^{M} \sum_{r=1}^{N} \frac{\sigma_{k}^{2}}{\sigma_{n}^{2}}\left(1-\cos \left(\frac{2 \pi}{\lambda} h_{1}\left(a_{k}+b_{r}\right)\right)\right)+\frac{1}{\sigma_{\beta}^{2}}\right]}\right\} \\
\times & \frac{\sqrt{\pi} \sigma_{\beta}}{4\left\{\frac{\sigma_{\beta}^{2}}{2}\left[\frac{T}{2} \sum_{k=1}^{M} \sum_{r=1}^{N} \frac{\sigma_{k}^{2}}{\sigma_{n}^{2}}\left(1-\cos \left(\frac{2 \pi}{\lambda} h_{1}\left(a_{k}+b_{r}\right)\right)\right)+\frac{1}{\sigma_{\beta}^{2}}\right]\right\}^{\frac{1}{2}}} .
\end{aligned}
$$

Similarly, it is straightforward to see that $\eta\left(\mathbf{h}_{1},-\mathbf{h}_{2}\right)=\eta\left(-\mathbf{h}_{1},-\mathbf{h}_{2}\right)$ which, together with (24), lead to $\mathbf{G}_{12}=0$. We also have $\mathbf{G}_{21}=0, \mathbf{G}_{13}=0, \mathbf{G}_{31}=0, \mathbf{G}_{23}=0$, and $\mathbf{G}_{32}=0$. Therefore, the matrix $\mathbf{G}$ is 
diagonal with elements given by

$$
\begin{aligned}
\mathbf{G}_{11}= & \frac{\frac{2-h_{1}}{2}-\frac{1-h_{1}}{\frac{\sigma_{\beta}^{2}}{\sigma_{n}^{2}} \frac{1}{2} T \sum_{k=1}^{M} \sum_{r=1}^{N} \sigma_{k}^{2}\left[1-\cos \left(\frac{4 \pi}{\lambda} h_{1}\left(a_{k}+b_{r}\right)\right)\right]+1}}{\left[\frac{2-h_{1}}{2} \frac{1}{\frac{\sigma_{\beta}^{2}}{\sigma_{n}^{2}} \frac{1}{2} T \sum_{k=1}^{M} \sum_{r=1}^{N} \sigma_{k}^{2}\left[1-\cos \left(\frac{2 \pi}{\lambda} h_{1}\left(a_{k}+b_{r}\right)\right)\right]+1}\right]^{2}} \\
\mathbf{G}_{22}= & 2 \frac{1-\exp \left(-h_{2}^{2} N T \frac{\sum_{k=1}^{M} \sigma_{k}^{2}}{\sigma_{n}^{2}}\right) \exp \left(-\frac{h_{2}^{2}}{\sigma_{\beta}^{2}}\right)}{\exp \left(-\frac{1}{2} h_{2}^{2} N T \frac{\sum_{k=1}^{M} \sigma_{k}^{2}}{\sigma_{n}^{2}}\right) \exp \left(-\frac{h_{2}^{2}}{2 \sigma_{\beta}^{2}}\right)} \\
\mathbf{G}_{33}= & 2 \frac{1-\exp \left(-h_{3}^{2} N T \frac{\sum_{k=1}^{M} \sigma_{k}^{2}}{\sigma_{n}^{2}}\right) \exp \left(-\frac{h_{3}^{2}}{\sigma_{\beta}^{2}}\right)}{\exp \left(-\frac{1}{2} h_{3}^{2} N T \frac{\sum_{k=1}^{M} \sigma_{k}^{2}}{\sigma_{n}^{2}}\right) \exp \left(-\frac{h_{3}^{2}}{2 \sigma_{\beta}^{2}}\right)}
\end{aligned}
$$

Thanks to the definition of $f\left(\varphi, h_{1}\right)$ in (14), $\mathbf{G}_{11}$ can be rewritten as in (13). Using all the assumptions and results above, the closed-form expression of the matrix WWB can be written as

$$
\mathbf{W W B}=\operatorname{Diag}\left(\sup _{h_{1}} \frac{h_{1}^{2}}{\mathbf{G}_{11}}, \sup _{h_{2}} \frac{h_{2}^{2}}{\mathbf{G}_{22}}, \sup \frac{h_{3}^{2}}{h_{3}}\right),
$$

with $\mathbf{G}_{11}, \mathbf{G}_{22}, \mathbf{G}_{33}$ given in (13), (28), and (29), respectively.

Note that in the original form of the Weiss-Weinstein bound (5), we have to optimize the matrix WWB w.r.t. $h_{1}, h_{2}$, and $h_{3}$. Since $\mathbf{G}_{i i}$ depends only on $h_{i}$, the optimization task is reduced to $\sup _{h_{k}} \frac{h_{k}^{2}}{\mathbf{G}_{k k}}, \forall k$ which is more tractable. Next, we give a closed-form expression for $\sup _{h_{k}} \frac{h_{k}^{2}}{\mathrm{G}_{k k}}, k=2,3$,

$$
\mathbf{W W B}_{k k}=\sup _{x \geq 0} \frac{x \exp (-a x)}{2[1-\exp (-2 a x)]}, a>0
$$

where $x=h_{k}^{2}$ and $a=N T \frac{\sum_{k=1}^{M} \sigma_{k}^{2}}{2 \sigma_{n}^{2}}+\frac{1}{2 \sigma_{\beta}^{2}}$. If we denote $g(x)=\frac{x \exp (-a x)}{2[1-\exp (-2 a x)]}$, then

$$
\frac{d g(x)}{d x}=\frac{\exp (-a x)[1-a x-\exp (-2 a x)-a x \exp (-2 a x)]}{4[1-\exp (-2 a x)]^{2}},
$$

which is always negative $\forall x>0$. Consequently, $g(x)$ is a monotonically decreasing function and has its maximum at $x=0$. Finally, we obtain:

$$
\sup _{h_{k}} \frac{h_{k}^{2}}{\mathbf{G}_{k k}}=\frac{1}{2\left(N T \frac{\sum_{k=1}^{M} \sigma_{k}^{2}}{\sigma_{n}^{2}}+\frac{1}{\sigma_{\beta}^{2}}\right)}, k=2,3,
$$

which concludes the proof. 


\section{References}

[1] E. Fishler, A. Haimovich, R. Blum, L. Cimini, D. Chizhik, and R. Valenzuela, "MIMO radar: an idea whose time has come," in Proc. of the IEEE Int. Conf. on Radar, 2004, pp. 71-78.

[2] J. Li and P. Stoica, MIMO Radar Signal Processing. New York: Wiley, 2009.

[3] J. Li, P. Stoica, L. Xu, and W. Roberts, "On parameter identifiability of MIMO radar," IEEE Signal Processing Lett., no. 14, pp. 968-971, Dec. 2007.

[4] L. Xu, J. Li, , and P. Stoica, "Target detection and parameter estimation for MIMO radar systems," IEEE Trans. Aerosp. Electron. Syst., no. 44, pp. 927-939, 2008.

[5] H. Godrich, A. Haimovich, and R. Blum, "Cramér-Rao bound on target localization estimation in MIMO radar systems," in Conf. Information Sciences and Systems, (CISS 08), 2008, pp. 134-139.

[6] Q. He, R. Blum, H. Godrich, and A. Haimovich, "Cramér-Rao bound for target velocity estimation in MIMO radar with widely separated antennas," in Conf. Information Sciences and Systems, (CISS 08), 2008, pp. 123-127.

[7] J.Li, L. Xu, P. Stoica, K. W. Forsythe, and D. W. Bliss, "Range compression and waveform optimization for MIMO radar: A Cramér-Rao bound based study," IEEE Trans. Signal Processing, vol. 56, no. 1, pp. 218-232, Jan. 2008.

[8] R. Boyer, "Performance bounds and angular resolution limit for the moving co-located mimo radar," IEEE Trans. Signal Processing, 2011 to appear.

[9] J. Tabrikian, "Barankin bounds for target localization for MIMO radars," in Proc. 4th IEEE Workshop on Sensor Array and Multi-channel Processing, Jul. 2006, pp. 278-281.

[10] J. Ziv and M. Zakai, "Some lower bounds on signal parameter estimation," IEEE Trans. Inform. Theory, vol. 15, pp. 386-391, May 1969.

[11] K. Bell, Y. Steinberg, Y. Ephraim, and H. L. V. Trees, "Extended Ziv-Zakai lower bound for vector parameter estimation," IEEE Trans. Signal Processing, vol. 43, pp. 624-638, Mar. 1997.

[12] B. Z. Bobrovsky and M. Zakai, "A lower bound on the estimation error for certain diffusion processes," IEEE Trans. Inform. Theory, vol. 22, pp. 45-52, Jan. 1976.

[13] A. Renaux, P. Forster, P. Larzabal, and C. Richmond, "The Bayesian Abel bound on the mean square error," in Proc. IEEE Int. Conf. Acoust., Speech, Signal Processing, Toulouse, FR, May 2006.

[14] A. J. Weiss and E. Weinstein, "A lower bound on the mean square error in random parameter estimation," IEEE Trans. Inform. Theory, vol. 31, pp. 680-682, Sep. 1985.

[15] I. Rapoport and Y. Oshman, "Weiss-Weinstein lower bounds for Markovian systems. part 1: Theory," IEEE Trans. Signal Processing, vol. 55, no. 5, pp. 2016-2030, May 2007.

[16] — - "Weiss-Weinstein lower bounds for Markovian systems. part 2: Applications to fault tolerant filtering," IEEE Trans. Signal Processing, vol. 55, no. 5, pp. 2031-2042, May 2007.

[17] A. Renaux, P. Forster, and P. Larzabal, "A new derivation of the Bayesian bounds for parameter estimation," in Proc. of IEEE Statistical Signal Processing Workshop - SSP05, Bordeaux, FR, Jul. 2005.

[18] A. Renaux, P. Forster, P. Larzabal, C. Richmond, and A. Nehorai, "A fresh look at the Bayesian bounds of the WeissWeinstein family," IEEE Trans. Signal Processing, vol. 56, no. 11, pp. 5334-5352, 2008.

[19] E. Weinstein and A. J. Weiss, "A general class of lower bounds in parameter estimation," IEEE Trans. Inform. Theory, vol. 34, pp. 338-342, Mar. 1988.

[20] W. Xu, "Performances bounds on matched-field methods for source localization and estimation of ocean environmental parameters," Ph.D. dissertation, Massachusetts Institute of Technology, Cambridge, MA, Jun. 2001.

[21] H. Nguyen and H. L. V. Trees, "Comparison of performance bounds for doa estimation," in IEEE 7th SP Workshop on SSAP, Jun. 1994, pp. 313-316. 


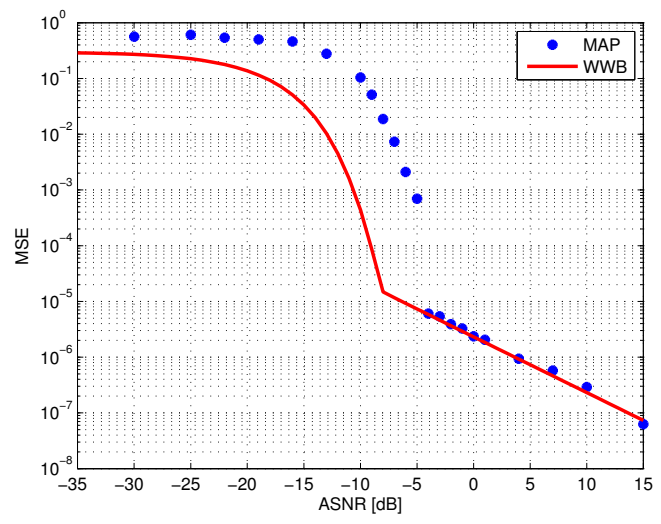

Figure 1: MAP estimator empirical global MSE and WWB of $u$ versus SNR

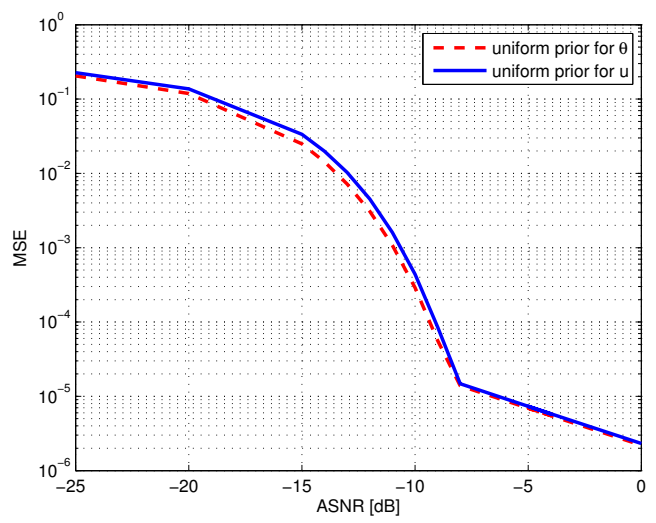

Figure 2: WWB of $u$ with a uniform prior pdf for $u$ and a uniform prior pdf for $\theta$ versus SNR

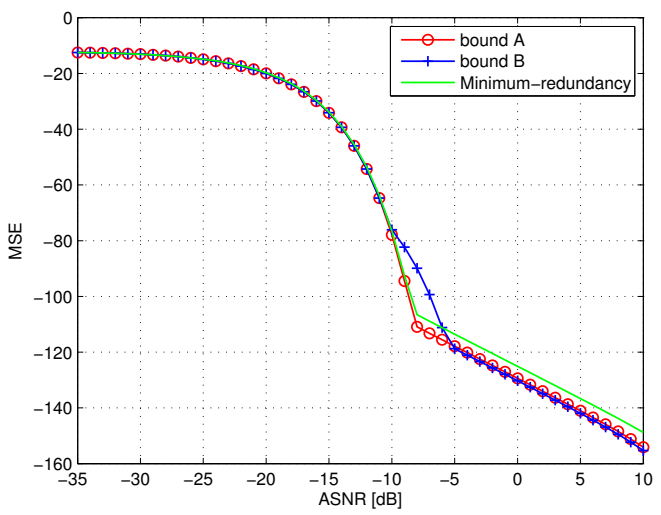

Figure 3: Receiver geometry investigation: WWB for the the lowest-threshold-point, the lowest-asymptotic-region, and the minimum-redundancy arrays. 